\title{
Can ultrasonography make identification of asymptomatic hyperuricemic individuals at risk for developing gouty arthritis more crystal clear?
}

\author{
Naomi Schlesinger* \\ See related research by Pineda et al., http://arthritis-research.com/content/13/1/R4
}

\begin{abstract}
Hyperuricemia is the most important risk factor for gouty arthritis. The quandary is how to predict which patient with asymptomatic hyperuricemia will develop gouty arthritis. Can ultrasonography help identify hyperuricemic individuals at risk for developing gouty arthritis? In the previous issue of Arthritis Research \& Therapy, Pineda and colleagues found ultrasonography changes suggestive of gouty arthritis in 25\% of hyperuricemic individuals. These were found exclusively in hyperuricemic individuals but not in normouricemic patients. Ultrasonography may serve as a noninvasive means to diagnose gouty arthritis in hyperuricemic individuals who have yet to develop symptomatic gouty arthritis.
\end{abstract}

In the previous issue of Arthritis Research \& Therapy, Pineda and colleagues present an interesting study evaluating the use of ultrasonography (US) to help identify hyperuricemic individuals at risk for gouty arthritis [1]. Hyperuricemia is the most important risk factor for gouty arthritis. The number of adults with hyperuricemia and gouty arthritis is increasing.

The National Health and Nutrition Examination Survey (NHANES) data from 2007 to 2008 showed a hyperuricemia (serum urate $\geq 7 \mathrm{mg} / \mathrm{dl}$ ) prevalence of $21.1 \%$ in men and $4.7 \%$ in women [2]. Most individuals with hyperuricemia, however, do not develop gouty arthritis [3]. The reported gouty arthritis prevalence in the 2007 to 2008 NHANES data was $5.9 \%$ in men and $2 \%$ in women,

*Correspondence: schlesna@umdnj.edu

Division of Rheumatology, Department of Medicine, UMDNJ - Robert Wood Johnson Medical School, MEB 468, One Robert Wood Johnson PI., P.O. Box 19, New Brunswick, NJ 08903-0019, USA with an overall prevalence of 3.9\% (8.3 million adults) [4]. The risk of developing gouty arthritis is dependent on the severity of hyperuricemia. In the Normative Aging Study, healthy patients with serum urate levels $\geq 9 \mathrm{mg} / \mathrm{dl}$ upon entry into the study had a cumulative incidence of acute flares that reached $22 \%$ after 5 years, whereas those with serum urate levels $\leq 7 \mathrm{mg} / \mathrm{dl}$ had an annual incidence of only $0.5 \%$ [5]. In yet another study, the 5 -year prevalence of gouty arthritis was $30 \%$ in individuals with serum urate levels $>10 \mathrm{~g} / \mathrm{dl}$ [6]. These numbers correlate with the recently reported NHANES data.

The quandary is how to predict which patient with asymptomatic hyperuricemia will develop gouty arthritis, and thus who will benefit from-long term anti-inflammatory and urate-lowering therapy. Serum urate levels and gouty arthritis prevalence are related to genetic variations in the SLC2A9, ABCG2 and SLC17A3 genes. Dehghan and colleagues developed a risk score based on variations of these three genetic loci. They suggested that their genetic risk score is associated with up to a 40-fold increased risk of developing gouty arthritis, suggesting that knowledge of the genotype may help identify hyperuricemic individuals at risk for developing gouty arthritis [7]. Can US serve as another potential method to help identify hyperuricemic individuals at risk for developing gouty arthritis?

Over the past several years, there has been a growing interest in musculoskeletal US in rheumatology. US visualizes tissues as acoustic reflections. Crystalline material reflects US waves more strongly than the surrounding tissues, such as unmineralized hyaline cartilage or synovial fluid. This enables distinction of monosodium urate (MSU) crystal deposition from the less echogenic surrounding soft tissues. MSU crystals are found in cartilage, tendon sheaths, synovial fluid and subcutaneous tissue. US detects deposition of MSU crystals on cartilaginous surfaces, as well as tophaceous material and typical erosions. A hyperechoic, irregular band over the superficial margin of the articular cartilage - described 
as a double contour sign or icing - is found exclusively in gouty arthritis [8] and represents crystalline precipitates of MSU. In addition, the presence of hypoechoic to hyperechoic inhomogeneous material surrounded by a small anechoic rim, representing tophaceous material and erosions adjacent to tophaceous material on US, are suggestive of the diagnosis of gouty arthritis. US is superior in detecting changes of gouty arthritis compared with other imaging modalities (magnetic resonance imaging, plain X-ray scans, computed tomography and three-dimensional rendering imaging) [9].

Pineda and colleagues support previous evidence that US may be useful in detecting gouty arthritis in hyperuricemic patients [1]. Puig and colleagues reported that $34 \%(n=12)$ of their asymptomatic hyperuricemic individuals had findings suggestive of tophaceous deposits [10]. Pineda and colleagues also studied a larger cohort in a controlled fashion [1]. US images of the most commonly affected joints - knees, ankles and first metatarsophalangeals - were obtained. The double contour sign and tophi were seen ultrasonographically in the knee hyaline cartilage and the first metatarsophalangeals. Tendinous infiltrations of tophaceous material were also observed. Interestingly, tendinous tophi and enthesopathies were not a rare finding in these patients. US changes suggestive of gouty arthritis were found in 25\% of hyperuricemic individuals. These changes were found exclusively in the hyperuricemic individuals but not in their control group of normouricemic individuals. The main limitation of both Puig and colleagues' study [10] and Pineda and colleagues' study [1] is that the US findings suggestive of gouty arthritis, tophi and the double contour sign were not proven MSU crystals. In both studies, therefore, a definite diagnosis of gouty arthritis was not established.

Whether finding sonographic evidence suggestive of gouty arthritis prior to development of acute flares will influence our decision of when to initiate and commit to a long-term urate-lowering therapy and chronic antiinflammatory treatment is still to be determined. US may serve as a noninvasive means to diagnose gouty arthritis in hyperuricemic individuals who have yet to develop symptomatic gouty arthritis. How long hyperuricemia must be present before MSU crystal deposition can be seen sonographically is currently not known. Future large, prospective, randomized controlled trials of patients with proven MSU crystal gouty arthritis are needed to further evaluate the use of US to predict the presence of asymptomatic gouty arthritis in an individual hyperuricemic patient.

\section{Abbreviations}

MSU, monosodium urate; NHANES, National Health and Nutrition Examination Survey; US, ultrasound.

\section{Competing interests}

The author declares that she has no competing interests.

\section{Published: 19 April 2011}

\section{References}

1. Pineda C, Amezcua-Guerra LM, Solano C, Rodriguez-Henriquez P, HernandezDiaz C, Vargas A, Hofmann F, Gutierrez M: Joint and tendon subclinical involvement suggestive of gouty arthritis in asymptomatic hyperuricemia: an ultrasound controlled study. Arthritis Res Ther 2011, 13:R4.

2. Zhu Y, Pandya B, Choi H: Prevalence of hyperuricemia in the US general population: the National Health and Nutrition Examination Survey (NHANES) 1999-2008 [abstract]. Arthritis Rheum 2010, 62(10, Suppl):S1363.

3. Zhang W, Doherty M, Pascual E, Barskova V, Guerne PA, Jansen TL, Leeb BF, Perez-Ruiz F, Pimentao J, Punzi L, Richette P, Sivera F, Uhlig T, Watt I, Bradin T: EULAR evidence based recommendations for gout. Part I: diagnosis. Report of a task force of the standing committee for international clinical studies including therapeutics (ESCISIT). Ann Rheum Dis 2006, 65:1301-1311.

4. Zhu Y, Pandya B, Choi H: Increasing gout prevalence in the US over the last two decades: the National Health and Nutrition Examination Survey (NHANES) [abstract]. Arthritis Rheum 2010, 62(10, Suppl):S2154.

5. Campion EW, Glynn RJ, DeLabry LO: Asymptomatic hyperuricemia. Risks and consequences in the Normative Aging Study. Am J Med 1987 82:421-426.

6. Agudelo C, Wise CM: Crystal-associated arthritis. Clin Geriatr Med 1998, 14:495-513.

7. Dehghan A, Köttgen A, Yang Q, Hwang SJ, Kao WL, Rivadeneira F, Boerwinkle E, Levy D, Hofman A, Astor BC, Benjamin EJ, van Duijn CM, Witteman JC, Coresh J, Fox CS: Association of three genetic loci with uric acid concentration and risk of gout: a genomewide association study. Lancet 2008, 372:1953-1961.

8. Thiele R, Schlesinger N: Diagnosis of gout by ultrasound. Rheumatology 2007, 46:1116-1121.

9. Thiele RG, Anandarajah AP, Tabechian D, Schlesinger N: Comparing the use of ultrasonography, magnetic resonance imaging, conventional radiography high-resolution $\mathrm{CT}$ scanning and 3-dimensional rendering in patients with crystal proven gout [abstract]. Ann Rheum Dis 2008, 67(Suppl II):248.

10. Puig JG, de Miguel E, Castillo MC, Rocha AL, Martínez MA, Torres RJ: Asymptomatic hyperuricemia: impact of ultrasonography. Nucleosides Nucleotides Nucleic Acids 2008, 27:592-595.

doi:10.1186/ar3290

Cite this article as: Schlesinger N: Can ultrasonography make identification of asymptomatic hyperuricemic individuals at risk for developing gouty arthritis more crystal clear? Arthritis Research \& Therapy 2011, 13:107. 